\title{
Effect of Oscillatory Motion of a Visco- Elastic Dusty Fluid Passes Through a Porous Medium Under the Presence of Magnetic Field
}

\author{
Ashok Kumar ${ }^{\# 1}$ and M.Lal ${ }^{{ }^{2}}$ \\ Department of Mathematics \\ ${ }^{1}$ BSAITM Faridabad-121005(India), ${ }^{2}$ MVN University Palwal-121105 (India) \\ ${ }^{\# 1}$ Ashok2bahin@gmail.com, ${ }^{* 2}$ dr.lalmange@gmail.com
}

\begin{abstract}
In this paper, we discuss the effect of oscillatory motion of a visco-elastic dusty fluid passes through a porous medium under the presence of magnetic field. Solid and spherical non conducting dust particles are distributed symmetrically. The approximate solutions are obtained for skin friction profile and velocity distribution for both the dusty particles and dusty liquid. Also study various parameters like $\lambda_{1}, \lambda_{2}, B, T$ etc. The results have been discussed graphically for different parameters.
\end{abstract}

Key words - Porous medium, oscillatory motion, MHD flow, parameters.

\section{INTRODUCTION}

Various researchers from various backgrounds like Engineering, Applied Science have shown interest on dusty visco-elastic fluid flow in various channels because of its uses in the field of engineering and environments science. Fluid such as oil, honey, blood and some polymer solution present with viscous and elastic properties such type of fluid are refers as visco-elastic fluid. The flow of dusty fluid has its importance in many applications such as power plant, waste water treatment, petroleum transport etc. Flow fluid under the presence of magnetic field and transfer of heat occurs in magneto-hydro dynamics (MHD), accelerators, generators, and pump etc. Such type of fluid flow has used in plasma studies, nuclear reactor, geothermal energy extraction and in the field of aerodynamics. The fluid flow through porous medium has great import ants due to recovery of crude oil from the pores of reservoir rocks.

The equations of an viscous-elastic liquid motion have been discussed by Walter [1] between two co axial cylinders. Saffman [2] have been derived the equations of motion for a gas carrying dust particles and studied the stability of Laminar flow for dusty gas. Also the dust concentration effects and time relaxation, which measure the change of velocity of dust particles have also been studied. The Coquette flow of a dusty gas has been studied by Nag, Jana and Data [3] between two infinite parallel plates in this paper they discuss the velocity of dust particles be less than the fluid velocity if plate is acceleratory. Oscillatory flow on Free convection radiation under the effect of magnetic field passes through a porous medium under the constant suction velocity has been studied by El-Hakiem [4].

Saidu, Waziri, Roko and Musa [5] analyzed the convective dusty viscous flow with MHD effects under the uniform magnetic field and constant presser gradient. Also the effects of various parameters on the velocity are shown graphically. The free convective MHD flow of a dusty gas has been studied by Prakash, Kumar and Dwivedi [6] passes through a semi infinite plate when the velocity was exponentially studied decreasing with time. Subsequently Sharma, Sharma and Varshney [7] discuss the temperature, velocity, skin friction and concentration of a dusty fluid in an oscillating infinite porous flat plate under the presence of transverse magnetic field. Subsequently Chand, Singh and Sharma [8] studied about the effect of radiation and hall current on transfer of heat in MHD flow of dusty oscillating fluid in the porous medium subject to uniform suction. Recently Dey and Dhar [9] investigated the free convective dusty fluid passes through a porous media in the presence of inclined magnetic field. The result of visco-elastic dusty fluid of first order fluid which passes through a rectangular porous channel has been analyzed by Chaturvedi, Shrivastav and Ahamad [10].More recently Parkas , Makinde, Kumar and Dwivedi [11] studied about the combined effect of buoyancy force when the oscillatory dusty fluid flow when the channel filled with a porous media. Subsequently, the solution of hydro-magnetic dusty fluid flow with periodic pressure gradient and volume friction in a rotating channel has been analytically discussed by Dey[12]. The effect of dust particle on the flow of oscillatory unsteady eclectically conducting viscous fluid passes through rotating horizontal channels has also been discussed. The quantity of physical importance has also been discussed with graph for various parameters. An unsteady MHD flow and heat transfer has been studied more recently by Manjunatha, Gireesha and Bagewadi[13] and derived series solution for a rotating dusty fluid under the pressure of Hall current and radiation effect. In the present paper the numerical results like velocity of dusty liquid and dust particles, skin friction of dusty liquid and dust 
particles, Hartmann number, Nusselt number and temperature profile has been derived and represented graphically for practical importance.

\section{NOMENCLATURE}

$\mathrm{r} \rightarrow$ Radial coordinate

$\mathrm{P} \rightarrow$ Presser

$\rho \rightarrow$ Density of liquid

$v^{*} \rightarrow$ Velocity profile of dust particles

$\mathrm{u}^{*} \rightarrow$ Velocity profile for dusty liquid

$\mathrm{s}_{1} \rightarrow$ Skin friction profile of dusty liquid

$\mathrm{s}_{2} \rightarrow$ Skin friction profile of dust particle

$\mu \rightarrow$ Coefficient of viscosity

$\mathrm{M} \rightarrow$ MHD flow

$\lambda_{1} \rightarrow$ The Stress relaxation time for Dusty fluid

$\lambda_{2} \rightarrow$ The Stress relaxation time for Dust particles

$\mathrm{B} \rightarrow$ Magnetic Parameter

$\mathrm{T} \rightarrow$ Temperature of the Dusty fluid

\section{FORMULATION OF THE PROBLEM}

Let us consider a oscillatory motion of visco-elastic dusty liquid in porous medium between the plates is applied normal to the flow region under the effect of magnetic field. Let $\mathrm{u}^{*}$ be the liquid velocity and $\mathrm{v}^{*}$ be the particle velocity in the direction of $\mathrm{x}$-axis.

Then the equations of motions are

$$
\begin{aligned}
& \left(1+\lambda_{1} \frac{\partial}{\partial t}\right) \frac{\partial u^{*}}{\partial t}=v^{*}\left(1+\lambda_{1} \frac{\partial}{\partial t}\right) \frac{\partial^{2} u^{*}}{\partial y^{2}}+\frac{k N_{0}}{\rho}\left(1+\lambda_{1} \frac{\partial}{\partial t}\right)\left(u^{*}-v^{*}\right)-M^{2} u^{*}-\frac{u^{*}}{K} \\
& m \frac{\partial v^{*}}{\partial t}=k\left(u^{*}-v^{*}\right)
\end{aligned}
$$

Let us introduce the following non-dimensional quantities

$$
y^{\prime}=\frac{y}{u_{0}}, u^{\prime}=\frac{u^{*}}{u_{0}}, v^{\prime}=\frac{v^{*}}{u_{0}}, t^{\prime}=\frac{v^{*} t}{u_{0}^{2}}, \eta^{\prime}=\frac{u_{0}^{2} \eta}{v^{*}}, k^{\prime}=\frac{k}{u_{0}^{2}}
$$

Using equation (3) in equation (1) and (2) and dropping dashes, we get

$$
\begin{aligned}
& \left(1+\lambda_{1} \frac{\partial}{\partial t}\right) \frac{\partial u^{*}}{\partial t}=\left(1+\lambda_{1} \frac{\partial}{\partial t}\right) \frac{\partial^{2} u^{*}}{\partial y^{2}}+\frac{1}{w}\left(1+\lambda_{1} \frac{\partial}{\partial t}\right)\left(u^{*}-v^{*}\right)-B^{2} u^{*}-\frac{u^{*}}{T} \\
& w \frac{\partial v^{*}}{\partial t}=\left(u^{*}-v^{*}\right)
\end{aligned}
$$

where

$$
\begin{array}{lll}
B^{2}=\frac{M^{2} u_{0}}{v^{*}} \quad, & T=\frac{v^{*} k}{u_{0}} \quad, \quad I=\frac{M N_{0}}{\rho} \\
w=\frac{M v^{*}}{k u_{0}^{2}} \quad, \quad \lambda_{1}=\lambda_{1} \quad, \quad \lambda_{2}=\frac{v^{*}}{u_{0}^{2}} \lambda_{2}
\end{array}
$$

Non dimensional boundary conditions are

$$
\begin{array}{ll}
u^{*}=u_{0} e^{-q t}, v^{*}=v_{0} e^{-q t} & \text { at } t=0 \\
u^{*}=v^{*}=0 & \text { at } t \rightarrow \infty
\end{array}
$$

Let us assume the solution of the $\mathrm{u}^{*}, \mathrm{v}^{*}$ in the form

$$
u^{*}=f(y) e^{-q t}
$$




$$
v^{*}=F(y) e^{-q t}
$$

The boundary conditions from equation(6) are:

$$
\begin{array}{ccc}
f=u_{0} & \text { at } & y=0 \\
f \rightarrow \infty & \text { at } & y \rightarrow \infty
\end{array}
$$

By using equations (7) and (8) the equations (4) and (5) becomes,

$$
\begin{aligned}
& \frac{\partial^{2}}{\partial y^{2}} f(y)-R^{2} f(y)=0, \\
& F(y)=\frac{f(y)}{1-w q},
\end{aligned}
$$

where

$$
R^{2}=\left[\frac{\left(1-\lambda_{1} q\right)\left[q+\frac{I q}{1-w q}\right]-B^{2}-\frac{1}{T}}{\left(\lambda_{2} q-1\right)}\right]
$$

From equation (9),$\quad\left(D^{2}-R^{2}\right) f(y)=0$

The complete solution of equation (11) be

$$
f(y)=C_{1} e^{R y}+C_{2} e^{-R y}
$$

Under boundary conditions (6), we get

$$
\begin{aligned}
& C_{1}=0 \text { and } C_{2}=u_{0} \text { so } \\
& f(y)=u_{0} e^{-R y}
\end{aligned}
$$

and

$$
F(y)=\frac{u_{0} e^{-R y}}{1-w q}
$$

So velocity component are

$$
\begin{aligned}
u^{*} & =u_{0} e^{-q t} e^{-R y}=u_{0} e^{-(q t+R y)} \\
v^{*} & =\frac{u_{0} e^{-(q t+R y)}}{1-w q}
\end{aligned}
$$

Skin Friction: The skin friction for dusty liquid and dust particles are obtained

$$
\begin{aligned}
& S_{1}=-\mu\left(\frac{\partial u^{*}}{\partial y}\right)_{y=0}=\mu R u_{0} e^{-q t} \\
& S_{2}=-\mu\left(\frac{\partial v^{*}}{\partial y}\right)_{y=0}=\frac{\mu}{1-w q} R u_{0} e^{-q t}
\end{aligned}
$$




\section{RESULT AND DISCUSSION}

Taking the value for $\mathrm{l}=0.40, \mathrm{w}=0.80, \mathrm{q}=0.20, \mathrm{t}=0.50, \mu_{0}=6$ and different value of $\mathrm{B}, \mathrm{T}, \lambda_{1}$ and $\lambda_{2}$, the variation of parameters shown graphically by figure 1 to 4 .

\begin{tabular}{|c|c|c|c|c|}
\hline Graph & $\mathrm{B}$ & $\mathrm{T}$ & $\lambda_{1}$ & $\lambda_{2}$ \\
\hline Data 1 & 1.10 & 5.10 & 0.22 & 0.55 \\
\hline Data 2 & 1.10 & 11 & 0.22 & 0.55 \\
\hline Data 3 & 2.50 & 21 & 0.55 & 0.80 \\
\hline Data 4 & 2.50 & 21 & 0.75 & 0.90 \\
\hline Data 5 & 3.50 & 26 & 1.50 & 2.50 \\
\hline Data 6 & 4.50 & 26 & 1.50 & 2.50 \\
\hline
\end{tabular}

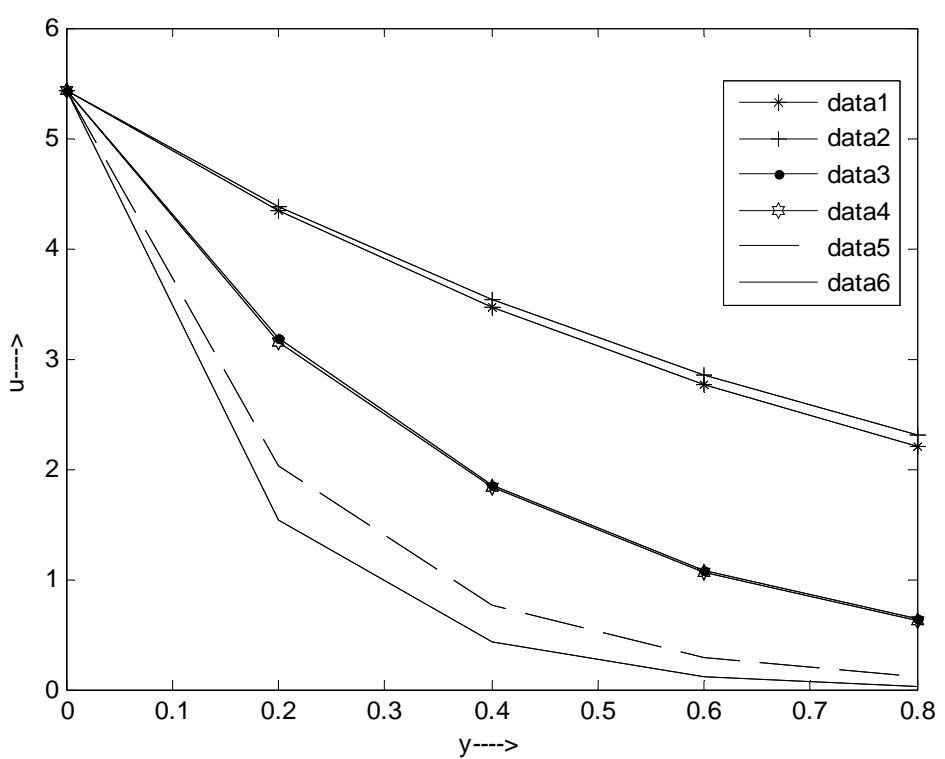

Fig.1 shows that the velocity profile of dusty liquid are increase when the value of $\mathrm{T}$ increase by data $1 \&$ data 2 , the velocity profile of dusty liquid are decrease when the value of B increase by data $5 \&$ data 6 , the velocity profile of dusty liquid are decrease when the value of $\lambda_{1}$ and $\lambda_{2}$ increase by data $3 \&$ data 4 .

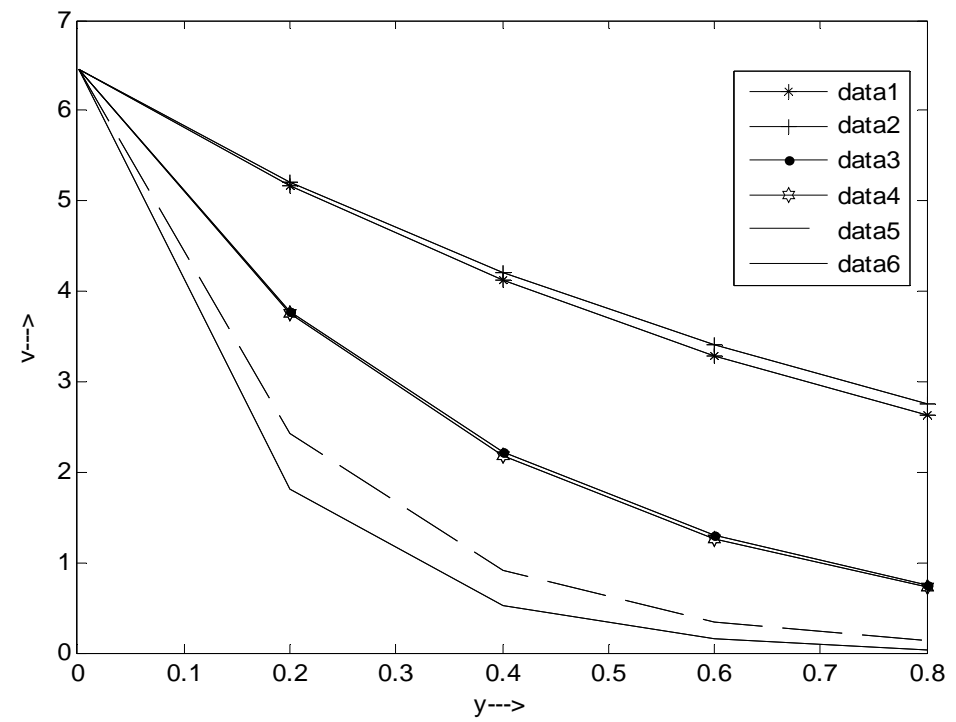

Fig. 2 shows that the velocity profile of dust particle are increase when the value of $\mathrm{T}$ increase by data $1 \&$ data 2 , the velocity profile of dust particle are decrease when the value of B increase by data $5 \&$ data 6 , the velocity profile of dust particle are decrease when the value of $\lambda_{1}$ and $\lambda_{2}$ increase by data $3 \&$ data 4 . 


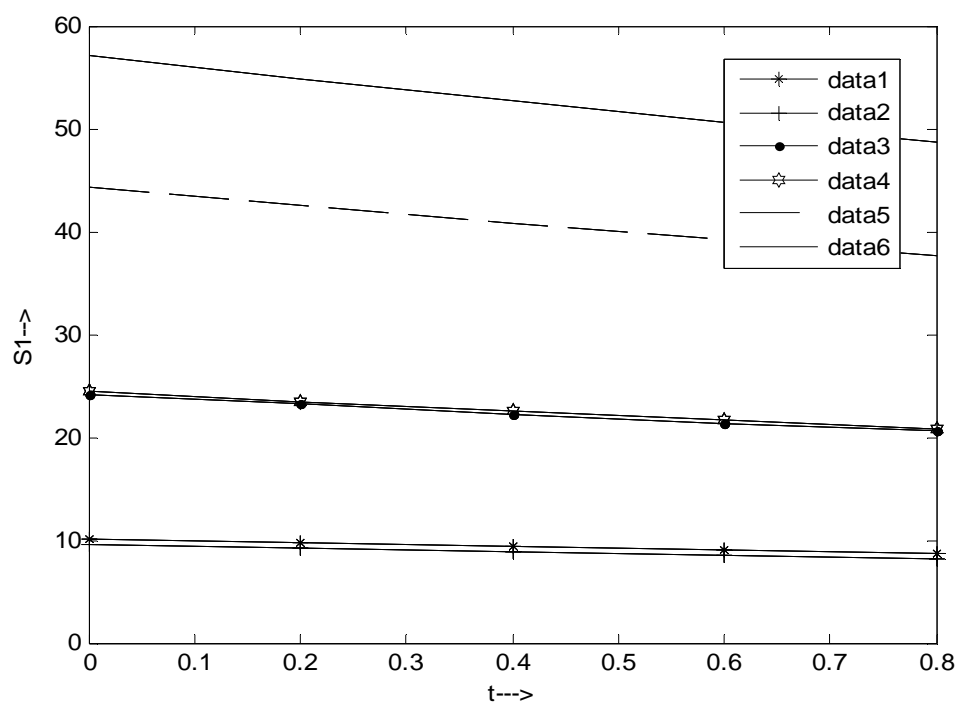

Fig.3shows that the skin friction of dusty liquid are decrease when the value of $\mathrm{T}$ increase by data $1 \&$ data 2 , the value of skin friction of dusty liquid are liquid are increase when the value of B increase by data $5 \&$ data 6 , the value of skin friction of dusty liquid are increase when the value of $\lambda_{1}$ and $\lambda_{2}$ increase by data $3 \&$ data 4 .

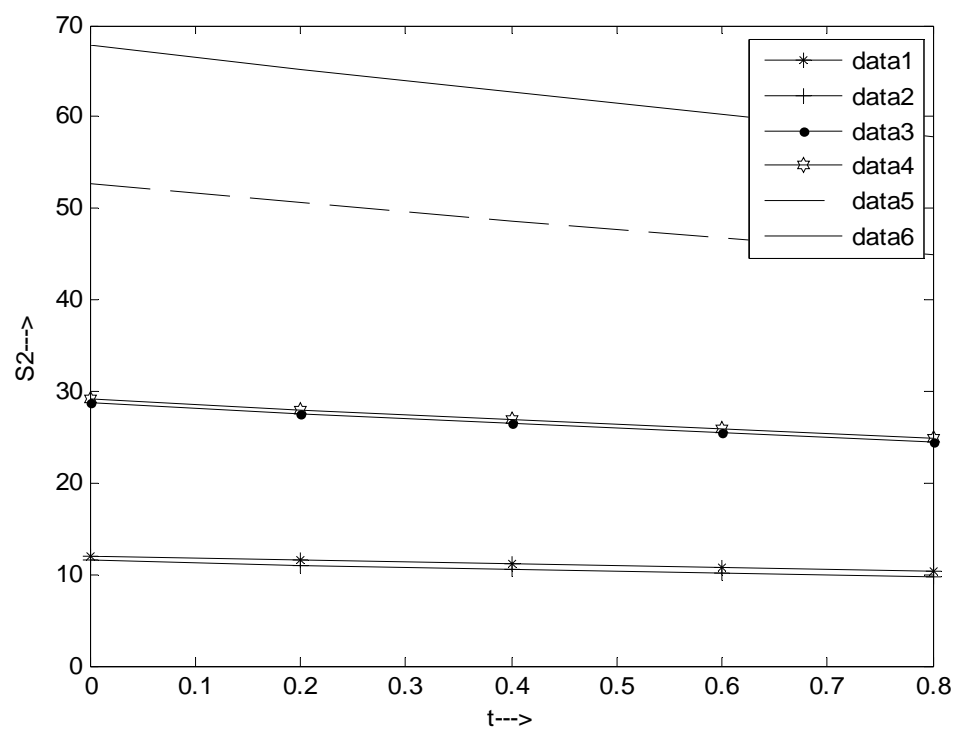

Fig.4shows that the skin friction of dust particle are decrease when the value of $\mathrm{T}$ increase by data $1 \&$ data 2 , the value of skin friction of dust particle are increase when the value of B increase by data $5 \&$ data 6 , the value of skin friction of dust particle are increase when the value of $\lambda_{1}$ and $\lambda_{2}$ increase by data $3 \&$ data 4 .

\section{CONCLUSION}

(1) Value of velocity profile of dust particle and dusty liquid are increase when value of $\mathrm{T}$ increase and the value of velocity profile of dust particle and dusty liquid are decrease when the value of $\mathrm{B}, \lambda_{1}$ and $\lambda_{2}$ increase.

(2) Value of skin friction of dusty liquid and dust particle are decrease when the value of $T$ increase and the value of skin friction of dusty liquid and dust particle are increase when the value of $B, \lambda_{1}$ and $\lambda_{2}$ decrease.

\section{REFERENCES}

[1] K.Walter, "The motion of an elastic-viscous liquid contained between co-axil cylinders", .Math., 13(4), Page 444-461 ,1960.

[2] P.G.Saffman, "On the stability of laminar flow of a dusty gas", Fluid Mech.,13, page 120-128,1962.

[3] S.K.Nag and R.N.Jana, "Couette flow of a dusty gas", Acta Mechanica, Springer verlag, 33, page 179-180,1979.

[4] M.A.El-Hakiem, "MHD oscillatory flow on free convection-radiation through a porous medium with constant suction velocity", Journal. of magnetism and magnetic materials, 220 , page 271-276,2000.

[5] Saidu Ibrahim, M.Y.Waziri, Roko Abubakar and Musa Himisu., "MHD effect on convective flow of dusty viscous fluid with volume fraction of dust particles", ARPN journal of engineering and applied science., 5(10), page 86- $91,2010$.

[6] Om Prakash, DevendraKumar and Y.K.Dwivedi, "MHD free convection flow of a visco-elastic (Kuvshiniski type) dusty gas through a semi infinite plate moving with velocity decreasing exponentially with time and radiative heat transfer", AIP Advances., 1 page 022132-1-022132-9,2011. 
[7] Sunil Kumar Sharma, Vineet Kumar Sharma and N.K.Varshney, "Effect of thermal diffusion MHD free convective flow of dusty oldroyd fluid through a porous medium bounded by an oscillating porous plate in slip flow regime with heat source/sink", International journal of Mathematical Archive.,3(11), page 4711-4717,2012.

[8] Khem Chand, K.D.Singh and Shavnam Sharma, "Effect of a hall current and rotation on heat transfer in MHD flow of oscillating dusty fluid in a porous channel", Indian Journal of pure and Applied Physics., 51, Page 669- 682,2013.

[9] Debasish Dey and Paban Dhar., "Free convective dusty visco-elastic fluid through a porous medium in the presence of inclined magnetic field and heat source/sink", Innovation International journal of Applied Research, 2(2),page 28-34,2014.

[10]Ruchi Chaturvedi, R.K.Shrivatav and Mohd. Salim Ahmad, "The numerical analysis of MHD flow of dusty visco-elastic first order oldroyd fluid when passing through a porous rectangular channel", International Journal of Modern Mathematical science, 9(1), page 46-57,2014.

[11]Om Prakash , O.D.Makinde, Devendra Kumar and Y.K Dwivedi., "Haet transfer to MHD oscillatory dusty fluid flow in a channel filled with a porous medium", Indian Academy of Sciences, 40(4), Page 1273-1282 2015.

[12]Devasish Dey, Hydro-Magnetic Oscillatory Dusty Fluid Flow with Volume Fraction and Periodic Pressure Gradient in a Rotating Channel, Int.J.Res.Appl.Sci.and Engng.Tech., 3(III), pp-869-877,2015.

[13]S.Manjunatha, B.J. Gireesha and C.S.Bagewadia, Series Solutions for an Unsteady flow and Heat Transfer of a Rotating Dusty Fluid with Radiation Effects, Acta Math. Univ. Comenianae,LXXXVI,1,,pp-111-125,2017.

\section{AUTHOURS PROFILE}

ASHOK KUMAR Education: M.Sc. Mathematics from M.D.U.Rohtak, PGDCA Kurukshetra University, M.Phil CDLU Sirsa and presently working as Assistant Professor in BSAITM, Faridabad.

MANGE.LAL Education: M.Sc. (Agra), Ph.D. (Kanpur), D.Sc.(Jabalpur), M.N.A.Sc. (Allahabad). Worked as different capacities i.e. Head of the Deptt. , Dean Faculty, Director in International reputed Universities of India. He has published 86 research papers in National and International reputed Journals. Appointed reviewer by American Mathematics society in 1984. Fifteen students awarded Ph.D. degree under his able guidance and also many students completed their M.Phil dissertation. His area of research be Solid Mechanics, Thermo -elasticity, Integral Equations and Series, Fluid Mechanics. He has 44 years of research and teaching experience. 